\title{
Salvage re-irradiation using stereotactic body radiation therapy for locally recurrent prostate cancer: the impact of castration sensitivity on treatment outcomes
}

\author{
Ron Lewin ${ }^{1 *} \mathbb{0}$, Uri Amit ${ }^{1}$, Menachem Laufer ${ }^{2}$, Raanan Berger ${ }^{3,4}$, Zohar Dotan ${ }^{2,4}$, Liran Domachevsky ${ }^{5}$,
} Tima Davidson ${ }^{5}$, Orith Portnoy ${ }^{6}$, Lev Tsvang ${ }^{1}$, Maoz Ben-Ayun ${ }^{1}$, Ilana Weiss ${ }^{1}$ and Zvi Symon ${ }^{1,4}$

\begin{abstract}
Background: Advances in imaging, biomaterials and precision radiotherapy provide new opportunities to salvage locally recurrent prostate cancer (PC). This study evaluates the efficacy and safety of re-irradiation using stereotactic body radiation therapy (SBRT). We hypothesized that patients with castrate-resistant PC (CRPC) would benefit less from local salvage.
\end{abstract}

Methods: A prospective clinical database was reviewed to extract 30 consecutive patients treated with prostate reirradiation. Gallium prostate specific membrane antigen (PSMA) ligand positron emission tomography was performed following prostate-specific antigen failure in all patients and biopsy was obtained in 18 patients (60\%). Re-irradiation was either focal $(n=13)$ or whole-gland $(n=17)$. Endo-rectal balloons were used in twenty-two patients and hydrogel spacers in eight patients. The median prescription dose was 5 fractions of 6.5 (range: 6-8) Gray (Gy).

Results: Median follow-up was 28 months. Failure occurred in 10 (out of 11) CRPC patients versus 6 (out of 19) castrate-sensitive patients ( $91 \%$ vs. 32\%, $p=0.008$ ) after a median of 13 and 23 months, respectively. Metastases occurred in $64 \%(n=7)$ of CRPC patients versus $16 \%(n=3)$ of castrate-sensitive patients $(p=0.007)$. Two patients experienced local in-field recurrence, thus local control was 93\%. The 2 and 3-year recurrence-free survival were $84 \%$ and $79 \%$ for castrate-sensitive patients versus 18\% and 9\% for CRPC patients ( $p<0.001)$, and 3-year metastasis-free survival was $90 \%$ versus $27 \%(p<0.01)$ for castrate-sensitive and CRPC patients, respectively. Acute grade II and III genitourinary (GU) toxicity occurred in 27\% and 3\%, and late GU toxicity in 30\% and 3\%, respectively. No $\geq$ grade II acute gastrointestinal (GI) toxicity occurred, and only one patient (3\%) developed late grade II toxicity.

Conclusions: Early delivery of salvage SBRT for local recurrence is associated with excellent 3-year disease control and acceptable toxicity in the castrate-sensitive phenotype. PSMA imaging for detection of local recurrence and the use of precision radiotherapy with rectal protective devices should be further investigated as a novel salvage strategy for radio-recurrent $P C$.

Keywords: Prostate, Salvage, Re-irradiation, Stereotactic body radiation therapy, Castrate resistant

*Correspondence: ron.lewin@sheba.health.gov.l

${ }^{1}$ Radiation Oncology Department, Sheba Medical Center, 52621 Ramat-Gan, Israel

Full list of author information is available at the end of the article

\section{Background}

Recurrence of PC after local definitive radiation therapy (RT) is a common clinical scenario. Up to a third of patients undergoing primary RT to the prostate will

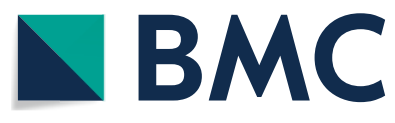

(c) The Author(s) 2021. Open Access This article is licensed under a Creative Commons Attribution 4.0 International License, which permits use, sharing, adaptation, distribution and reproduction in any medium or format, as long as you give appropriate credit to the original author(s) and the source, provide a link to the Creative Commons licence, and indicate if changes were made. The images or other third party material in this article are included in the article's Creative Commons licence, unless indicated otherwise in a credit line to the material. If material is not included in the article's Creative Commons licence and your intended use is not permitted by statutory regulation or exceeds the permitted use, you will need to obtain permission directly from the copyright holder. To view a copy of this licence, visit http://creativecommons.org/licenses/by/4.0/. The Creative Commons Public Domain Dedication waiver (http://creativeco mmons.org/publicdomain/zero/1.0/) applies to the data made available in this article, unless otherwise stated in a credit line to the data. 
experience biochemical failure within 10 years [1-3]. The most common pattern of relapse for all risk groups is local recurrence within the prostate or seminal vesicles (SV) [4, 5]. In the absence of distant metastases, salvage local treatment may lead to prolonged disease control and possibly cure. There is no clear consensus on the optimal management of primary RT failure. Acceptable treatment options includes local salvage with radical prostatectomy, cryotherapy, and focal therapies such as high-intensity focused ultrasound (HIFU) [5]. However as these options are often associated with urinary and sexual morbidity, many patients are managed with lifelong androgen deprivation therapy (ADT) $[6,7]$, which has a major impact on quality of life [8], is not curative by itself, and eventually induces a more aggressive CRPC phenotype [9]. In recent years, re-irradiation using brachytherapy [10-14] has been included in guideline options and SBRT is under investigation [15-17].

Improvements in techniques of imaging to locate sites of recurrence and to guide biopsies or focal therapies have been considerable. PSMA-PET imaging and dynamic contrast enhanced magnetic resonance imaging (DCE-MRI) are useful tools for defining regions suspicious for recurrence [5, 18-21]. Furthermore, considerable advances in precise treatment planning and delivery, as well as the introduction of endo-rectal balloons (ERBs) $[22,23]$ and spacers [24-26] have improved the ability to offer safer re-irradiation in recurrent disease.

The purpose of this study is to report safety and efficacy outcomes of prostate re-irradiation using SBRT with contemporary planning and delivery techniques. In addition, we aim to stratify treatment outcomes according to castrate sensitivity status for better patient selection. We hypothesized that local salvage of castrate-sensitive versus CRPC patients would be associated with improved outcomes.

\section{Methods}

Patient data was extracted from our Institutional Review Board approved clinical database. Eligible patients were treated with salvage SBRT to the prostate following local failure of primary external-beam RT (EBRT) or brachytherapy. Patients with a history of severe late toxicity, such as radiation proctitis, cystitis, or urinary stricture following previous RT were not eligible for salvage SBRT. Patients were specifically asked about the occurrence and severity of such symptoms, and medical records were also scrutinized to identify events suggestive of grade 3 or 4 toxicity. In questionable cases, rectoscopy and cystoscopy were performed prior to re-irradiation. All patients had experienced a biochemical failure after primary RT defined as a rise in PSA $>2 \mathrm{ng} / \mathrm{ml}$ above nadir PSA value according to the Phoenix criteria. In order to study the effects of CRPC phenotype on outcomes, patients receiving salvage SBRT were categorized as harboring castrate-sensitive or castrate-resistant local recurrence. Castrate-sensitive patients had not received androgen ablation for 2 years prior to the recurrence and salvage SBRT. CRPC patients had all received androgen ablation following failure of primary radiation and were not initially referred for consideration of local salvage by their treating physicians. They subsequently developed a biochemical failure on ADT, were find to harbor local disease on imaging and then referred for salvage radiation. $\mathrm{Ga}^{68}$ PSMA PET imaging was performed in all patients prior to salvage SBRT, demonstrating at least a local pathological PSMA uptake. Histologic confirmation of local recurrence was the objective in all cases, but not always achieved due to patient or physician preference. All patients treated without a biopsy had very suggestive imaging on both PSMA PET and dynamic contrastenhanced MRI. Salvage re-irradiation was delivered with SBRT using volumetric modulated arc therapy (VMAT) (RapidArc, Varian medical systems. Palo Alto, Ca). Focal re-irradiation was the preferred strategy to reduce the risk of toxicity. Clinical target volume (CTV) was defined as the PSMA-avid lesion and the DCE-MRI lesion with a 3-5 mm margin for well-defined lateralized recurrences. Whole gland re-treatment was reserved for patients with large tumors crossing or abutting the midline or bilateral recurrences.

All patients had fiducial markers placed in the prostate. Hydrogel spacers are associated with lower anterior rectal wall dose than ERBs and thus were the preferred method for rectal displacement in patients with recurrence in the prostate gland and were implanted in patients who could afford the cost. ERBs were preferred for seminal vesicle recurrences, as the spacer is not as effective in this setting. Urethral-sparing dose painting was used to reduce urethral toxicity (Fig. 1). For urethra-sparing planning prescribed dose to the urethra planning risk volume (uPRV) was $32.5 \mathrm{~Gy}$ (6.5 Gy/ fraction), and dose constraints were $\mathrm{D} 98 \% \geq 30.875 \mathrm{~Gy}$ (95\% of 32.5 Gy) and D2\% $<35.75$ Gy (110\% of 32.5 Gy). Dose constraints for the rectal wall were V100\%<5\% (of the prescribed dose to the prostate), V90\% $<15 \%$, V80\% $<20 \%$, and V38 Gy $<2$ cc. Treatment was delivered with a full bladder in 5 fractions using image guidance with cone beam computed tomography (CBCT). Toxicity was reported using the common terminology criteria for adverse events (CTCAE) v4.0. Acute toxicity was defined as adverse reactions occurring within 3 months after salvage SBRT, and late toxicity for those occurring later than 3 months.

Median follow-up time was calculated from salvage SBRT till recurrence or death. RFS was defined as the 

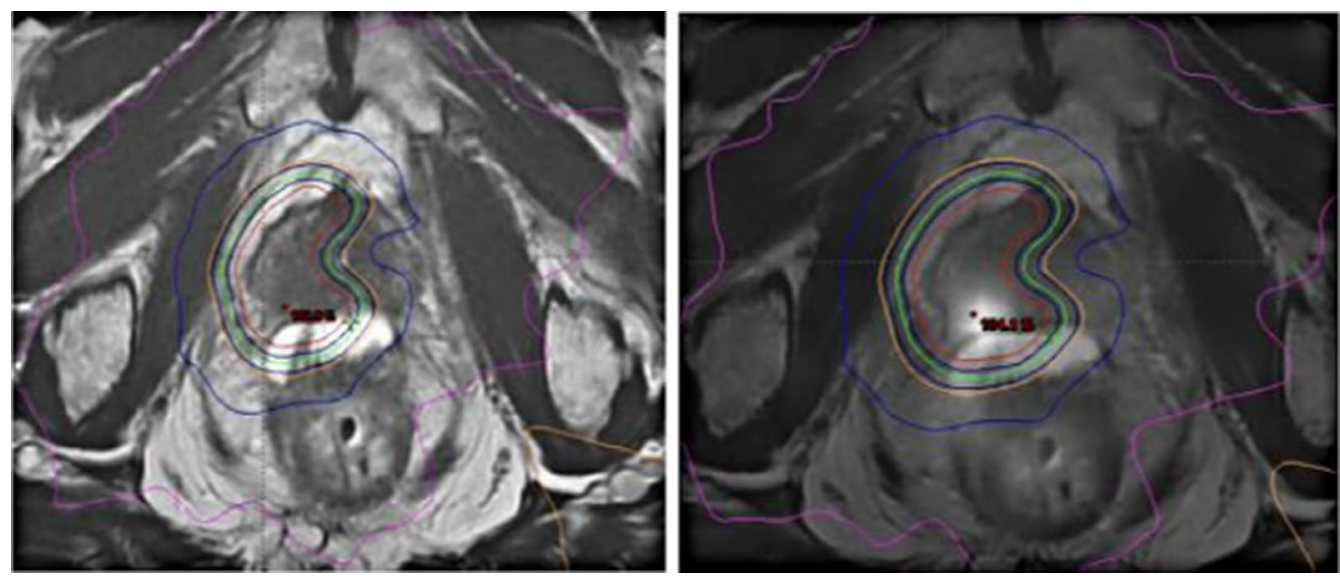

Fig. 1 An example of focal re-irradiation using PSMA-PET for target delineation, urethral dose sparing, and a hydrogel rectal spacer

length of time from salvage SBRT to either biochemical/ clinical failure or death from prostate cancer, and was estimated using the Kaplan-Meier method. Metastasisfree survival (MFS) was defined as the time from salvage SBRT to the date of onset of new distant metastases or when censored at last date. The log rank test was used to assess the difference between groups (SPSS Inc., IBM). P-value $<0.05$ was considered statistically significant. The difference in probability of local in-field recurrence after salvage SBRT between castrate-sensitive and CRPC patients was compared using the chi-square test.

\section{Results}

Thirty patients treated between 2015 and 2018 with reirradiation to the prostate following local failure were extracted from our database.

\section{Patient and treatment characteristics at initial diagnosis}

The median age at time of primary RT was 62 years (range: $52-75$ years). Patients were classified as low $(\mathrm{n}=7)$, intermediate $(\mathrm{n}=11)$, and high $(\mathrm{n}=12)$ risk according to the national comprehensive cancer network $(\mathrm{NCCN})$ risk stratification. Primary RT to the prostate was given either with EBRT $(n=25)$ to a median dose of 80 Gy (range: 74-82 Gy), or low dose rate (LDR) brachytherapy $(n=5)$ using $\mathrm{I}^{125}$ to a prescription dose of $145 \mathrm{~Gy}$. All high-risk patients and 5 intermediate risk patients received long and short course ADT, respectively, in conjunction with RT.

\section{Primary RT failure}

Median time from primary RT to biochemical/clinical failure was 72 months (range: 18-176 months). One third of patients $(n=11)$ received ADT-only as first salvage treatment upon failure for a median duration of
53 months (7-191 months) leading to the emergence of CRPC prior to their referral for local salvage treatment. $\mathrm{Ga}^{68}$ PSMA PET scans were performed in all patients prior to salvage SBRT. All patients had local pathological PSMA uptake: prostate-only in 18 patients, prostate and SV in 10 patients, and SV-only in 2 patients. Local staging at recurrence was stage $\geq \mathrm{T} 3$ in 14 patients (47\%). Regional (nodal) pathological uptake was also observed in 7 patients (23\%), one of whom had two small vertebral metastases. Biopsy confirming recurrent prostate adenocarcinoma was performed in 18 patients (60\%). Gleason score at recurrence was only reported in 8 of these cases, all in the high-risk category (Gleason 10 in one patient, Gleason 9 in six patients, and Gleason 8 in one patient).

\section{Patient and tumor characteristics prior to salvage SBRT}

Median interval from primary RT to re-treatment was 9 years (range 2-20 years). Histologic confirmation of recurrence was obtained in $60 \%$ of patients. Patient and tumor characteristics prior to salvage SBRT are detailed in Table 1.

The median salvage SBRT dose was 5 fractions of 6.5 Gy (range: 6-8 Gy) given every other day. Wholegland salvage SBRT was given in 17 patients $(57 \%)$ and focal salvage therapy in 13 (43\%) patients. Urethral sparing was achieved in 17 (57\%) patients (in 13 patients by focal therapy and in 4 receiving whole gland). Curative attempt radiotherapy was delivered to all disease sites in patients with PSMA uptake in pelvic nodes and in patients with oligometastases. Thus one patient with 2 oligo-metastases received SBRT to the vertebral lesions to a dose of 24 Gy in 3 fractions in addition to prostate reirradiation. Seven patients (23\%) with PSMA-avid lymph nodes also received nodal SBRT to a total dose of $25 \mathrm{~Gy}$ in 5 fractions. Rectal sparing techniques were utilized 
Table 1 Patient and tumor characteristics

\begin{tabular}{|c|c|c|c|}
\hline & All patients $(n=30)$ & Castrate-resistant $(n=11)$ & $\begin{array}{l}\text { Castrate- } \\
\text { sensitive } \\
(n=19)\end{array}$ \\
\hline $\begin{array}{l}\text { Age, years } \\
\text { Median (range) }\end{array}$ & $71(56-83)$ & $72(56-83)$ & $69(57-80)$ \\
\hline $\begin{array}{l}\text { Pre-salvage PSA, ng/ml } \\
\text { Median (range) }\end{array}$ & $3.63(0.05-77)$ & $3.16(0.05-12.8)$ & $4.1(0.65-77)$ \\
\hline \multicolumn{4}{|l|}{ Pre-salvage imaging, $n$ (\%) } \\
\hline $\mathrm{Ga}^{68}$ PSMA PET & $30(100 \%)$ & $11(100 \%)$ & $19(100 \%)$ \\
\hline mpMRI & $11(37 \%)$ & $4(36 \%)$ & $7(37 \%)$ \\
\hline \multicolumn{4}{|l|}{ Location of local recurrence, $n$ (\%) } \\
\hline Prostate-only & $18(60 \%)$ & $8(73 \%)$ & $10(53 \%)$ \\
\hline Prostate and SV & $10(33 \%)$ & $3(27 \%)$ & $7(37 \%)$ \\
\hline SV-only & $2(7 \%)$ & $0(0 \%)$ & $2(11 \%)$ \\
\hline \multicolumn{4}{|l|}{ Staging at recurrence, $n$ (\%) } \\
\hline T-stage $\geq T 3$ (locally advanced) & $14(47 \%)$ & $6(55 \%)$ & $8(42 \%)$ \\
\hline Node positive (regional) & $7(23 \%)$ & $3(27 \%)$ & $4(21 \%)$ \\
\hline Oligo-metastatic & $1(3 \%)$ & $1(9 \%)$ & $0(0 \%)$ \\
\hline \multicolumn{4}{|l|}{ Histologic confirmation, n (\%) } \\
\hline Yes & $18(60 \%)$ & $6(55 \%)$ & $12(63 \%)$ \\
\hline Median Gleason score ${ }^{1}$ (range) & $9(8-10)$ & & \\
\hline
\end{tabular}

Note that $83 \%$ of patients had high-risk features at recurrence (either castrate-resistant, Gleason $\geq 8$, T-stage $\geq T 3$, or node positive)

SBRT, stereotactic body radiation therapy; PSA, prostate specific antigen; mpMRI, multi-parametric magnetic resonance imaging; Ga ${ }^{68}$-PSMA, gallium-68 prostate specific membrane antigen positron; PET, positron emission tomography; SV, seminal vesicles

${ }^{1}$ Data was available for 8 patients

in all patients, ERB (Rectal Pro75, ELRAD International Ltd, International Warehouse, Dam Sluisweg 6, 1332 EC Almere) in 22 (73\%) patients, and hydrogel spacer (Boston Scientific Corp., Marlborough, Massachusetts) in 8 (27\%) patients. Short-term ADT was initiated with salvage SBRT in 15 out of 19 (79\%) patients with castratesensitive disease, while additional 11 CRPC patients continued long-term ADT concomitantly with salvage SBRT. Treatment characteristics are shown in Table 2.

\section{Treatment outcomes and toxicities}

Median follow-up following salvage SBRT was 28 months (range: 19-83 months). Biochemical / clinical failure post salvage SBRT occurred in 16 patients (53\%) after a median time of 16 months (range: 6-38 months). Ten of these patients (63\%) developed metastatic progression (bone and/or viscera), 3 had regional (nodal) failure, and 3 local failures (of which 2 were in-field recurrences). Thus, local control of salvage SBRT to the prostate for the entire cohort was $93 \%$. In subgroup analysis, failure occurred in 10 out of 11 CRPC patients versus 6 out of 19 castrate-sensitive patients (91\% vs. 32\%), $p=0.008$. Median time to failure in the CRPC group was 13 months (range: 6-28 months) compared to 23 months (range: 10-38 months) in the castrate-sensitive group.
Metastatic progression occurred in 7 out of 11 CRPC patients and in 3 out of 19 castrate-sensitive patients (64\% vs. $16 \%, p=0.007)$.

Five out of the 16 patients with disease recurrence had eventually died of their disease after a median interval of 28 months from salvage SBRT. Thirteen patients $(68 \%)$ in the castrate-sensitive group versus 1 patient (9\%) in the CRPC group were without evidence of disease after a median follow-up of 26 months (range: 16-43 months). Median RFS for the entire cohort was 22 months (range: 6-43 months). For castrate-sensitive versus CRPC patients, median RFS was 26 (range: 10-43) versus 13 months (range: $6-28$ ) months, $p<0.001$. The 2 and 3-year RFS for all patients were $60 \%$ and $53 \%$, with a significant difference between castrate-sensitive patients ( $84 \%$ and $79 \%$, respectively) versus CRPC patients (18\% and $9 \%$, respectively), $p<0.001$ (Fig. 2). Median MFS for the entire cohort was 25 months (range: 6-64 months). The 2 and 3-year MFS for the castrate-sensitive group versus the CRPC group were $90 \%$ versus $36 \%(p=0.001)$ at 2 years, and $90 \%$ versus $27 \%(p<0.001)$ at 3 years (Fig. 3).

Treatment outcomes are shown in Table 3.

Acute grade $2 \mathrm{GU}$ toxicity, primarily cystitis with frequency, urgency, and dysuria occurred in 8 patients 
Table 2 Treatment characteristics

\section{Target volume}

Whole gland therapy

$17(57 \%)$

$13(43 \%)$

$15(50 \%)$

$11(100 \%)$

$11(100 \%)$

$0(0 \%)$

$6(32 \%)$

Focal salvage therapy

LHRH agonist

Initiated with salvage SBRT (for 6 months)

Continued with salvage SBRT

2nd generation anti-androgen

Initiated with salvage SBRT

Continued with salvage SBRT ${ }^{1}$

Rectal sparing

Endo-rectal balloon

Hydrogel spacer

Urethral sparing

Yes

Prostate dose/fraction (Gy)

Median (range)

Number of fractions

Pelvic lymph nodes irradiation

Given in ${ }^{2}$
$22(73 \%)$

$8(27 \%)$

$17(57 \%)$

$6.5(6-8)$

5

7 (23\%)
1 (5\%)

1 (9\%)

7 (64\%)

15 (79\%)

$4(36 \%)$

$4(21 \%)$

$3(27 \%)$

14 (74\%)

$6.25(6-7.25)$

$6.5(6-8)$

5

$3(27 \%)$

$5(26 \%)$

SBRT, salvage stereotactic body radiation therapy; Gy, gray; LHRH, luteinizing hormone-releasing hormone

${ }^{1}$ Castrate-resistant patients

${ }^{2}$ Dose for nodal SBRT was 25 Gy in 5 fractions

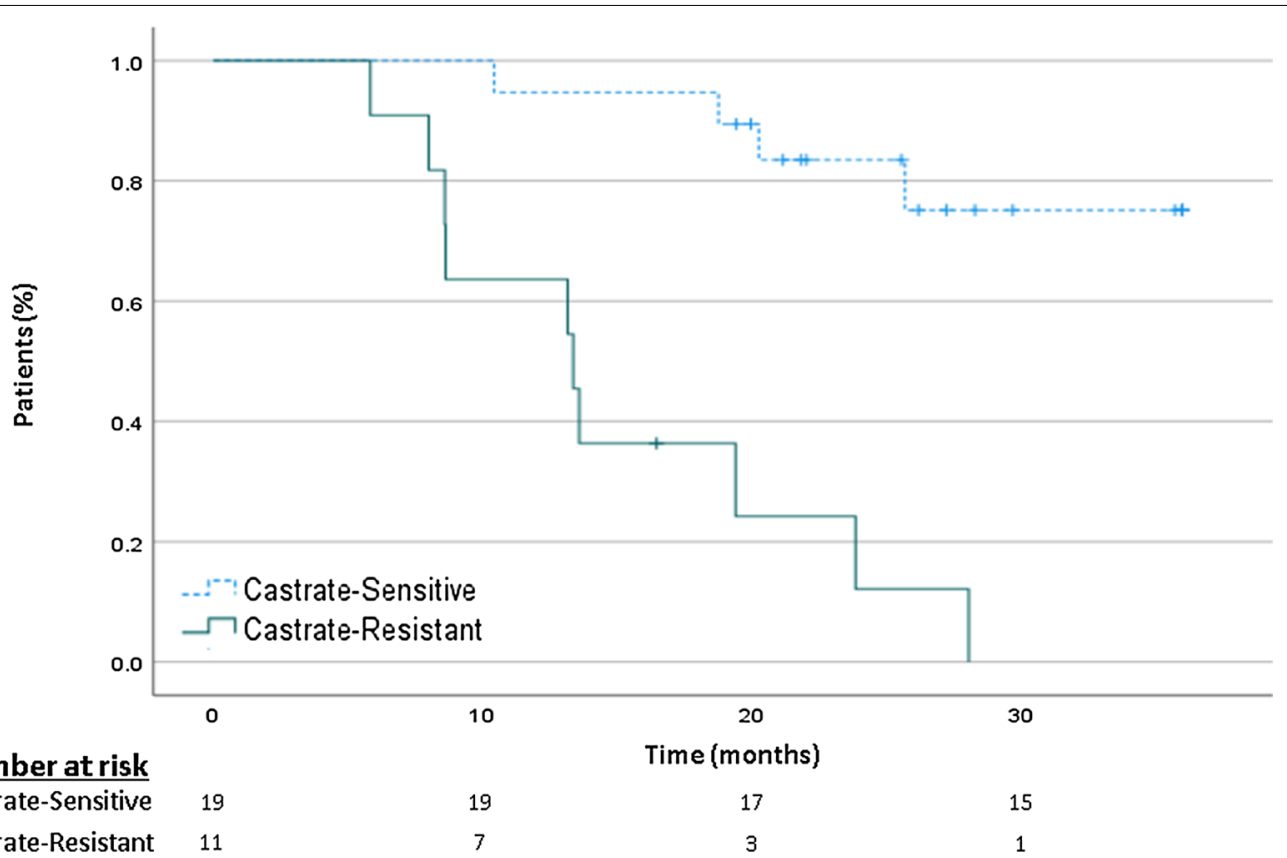

Fig. 2 Recurrence-free survival at 3 years 


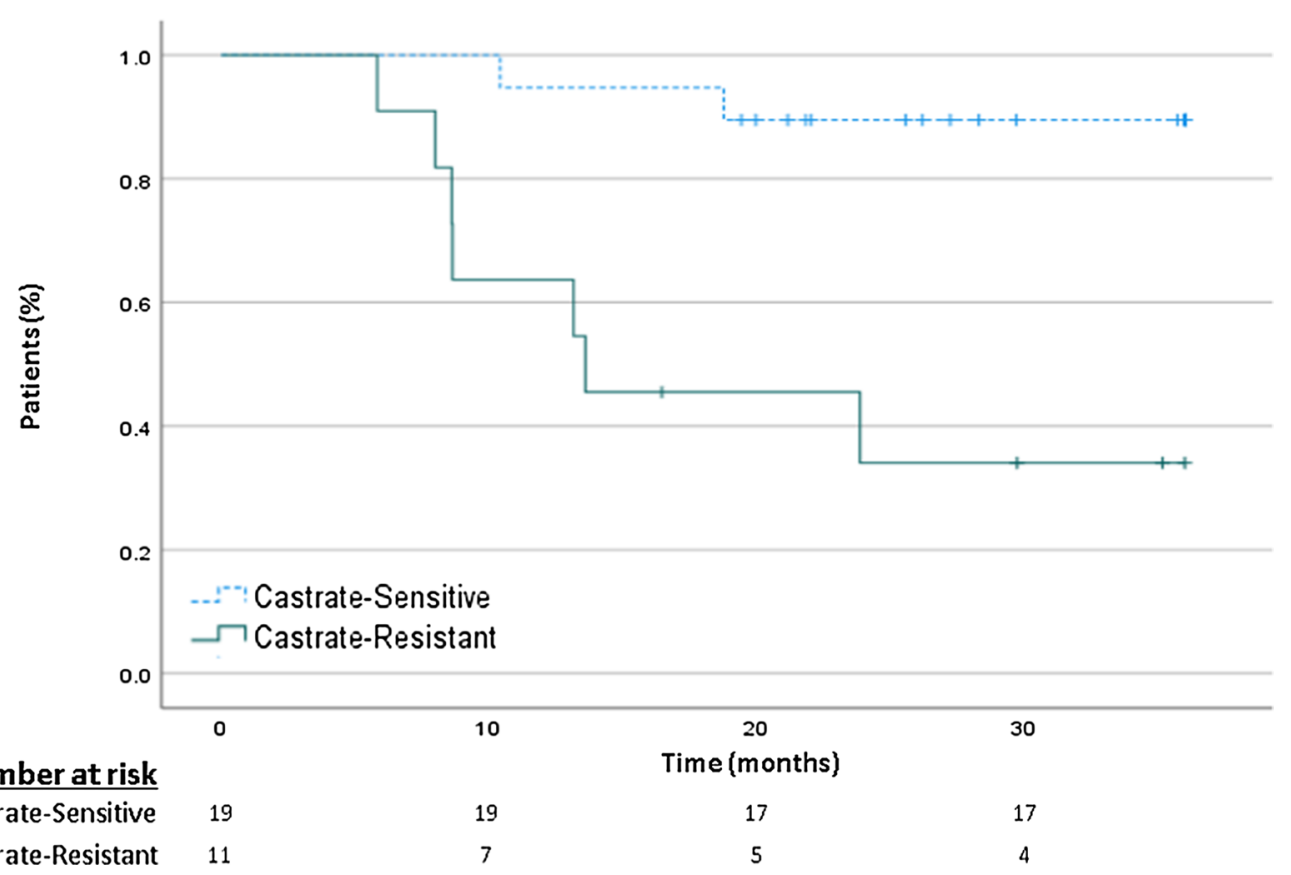

Fig. 3 Metastasis-free survival at 3 years

Table 3 Treatment outcomes

\begin{tabular}{|c|c|c|c|c|}
\hline & All patients $(n=30)$ & $\begin{array}{l}\text { Castrate-resistant } \\
(n=11)\end{array}$ & $\begin{array}{l}\text { Castrate-sensitive } \\
(\mathrm{n}=19)\end{array}$ & $p$-value \\
\hline Biochemical/clinical failure & $16(53 \%)$ & $10(91 \%)$ & $6(32 \%)$ & 0.008 \\
\hline Metastatic progression & $10(63 \%)$ & $7(64 \%)$ & $3(16 \%)$ & 0.007 \\
\hline Median time to failure, months (range) & $16(6-38)$ & $13(6-28)$ & $23(10-38)$ & \\
\hline Median RFS, months (range) & $22(6-43)$ & $13(6-28)$ & $26(10-43)$ & $<0.001$ \\
\hline 2 and 3-year RFS & $60 \%$ and $53 \%$ & $18 \%$ and $9 \%$ & $84 \%$ and $79 \%$ & $<0.001$ \\
\hline 2 and 3-year MFS & & $36 \%$ and $27 \%$ & $90 \%$ and $90 \%$ & 0.001 \\
\hline
\end{tabular}

RFS, recurrence-free survival; MFS, metastasis-free survival

(27\%), typically commenced mid-treatment and peaked during the first month after treatment and resolved in 3 patients by 3 months, yet persisted as late grade 2 $\mathrm{GU}$ toxicity in 5 patients. An additional 4 patients with hardly any early symptoms developed new onset late grade 2 cystitis $(n=2)$ and incontinence $(n=2)$, starting 7-9 months after treatment. Thus, late grade $2 \mathrm{GU}$ toxicity occurred in 9 patients (30\%). Acute grade 3 hematuria occurred in 1 patient (3\%) two months after completion of radiotherapy and required hospitalization and hemostasis, and 1 patient (3\%) developed a late grade 3 urethral stricture 10 months after completion of treatment. There was no $\geq$ grade 2 acute GI toxicity. Late grade $2 \mathrm{GI}$ incontinence occurred in 1 (3\%) patient who had suffered from grade 1 fecal incontinence prior to salvage SBRT. No late grade $3 \mathrm{GI}$ events occurred.
One patient developed late grade 3 osteomyelitis of the symphysis pubis 9 months following treatment.

\section{Discussion}

In this study we found that for patients with local failure after primary RT to the prostate, re-irradiation with salvage SBRT using rectal protection was associated with excellent local control (93\%) and negligible rectal toxicity. For patients with castrate-sensitive local recurrence, salvage SBRT was associated with a 3-year RFS of $80 \%$, suggesting a potential for long-term disease control and possibly cure in these patients. Despite excellent local control, most patients with castrate-resistant local recurrence rapidly developed distant metastases. Overall, about a half of our cohort were relapse free at the time of follow-up without androgen ablation. This was achieved despite the fact that most patients (83\%) had high-risk 
features at recurrence (either CRPC, T3/4 disease, regional nodal spread, or Gleason $\geq 8$ ). Treatment was well-tolerated with an acceptable rate of genitourinary toxicity and no severe rectal toxicity. We hypothesize this was achieved by meticulous treatment preparation, planning and delivery. This includes rectal protection by spacers or endo-rectal balloons, urethral sparing, imageguidance using fiducial markers, and PET/MRI-CT simulation fusions for accurate contouring and smaller margins.

Timing of salvage SBRT and the status of hormonal sensitivity seem to be crucial factors for success. Patients that were initially treated with systemic ADT at first recurrence and subsequently developed castrate-resistant disease prior to salvage SBRT were more prone to rapid development of distant metastases and had significantly poorer outcomes. These patients are presumed to harbor undetectable resistant micro-metastases at time of salvage SBRT with rapid progression to overt metastatic disease following treatment. We believe that salvage therapy should be initiated early in face of local failure, and implementation of novel imaging such as PSMA-PET scans at biochemical failure can improve early detection of local recurrence amendable to local salvage [27]. Early salvage can prevent the emergence of castrate-resistant tumor cells within the prostate, reduce the likelihood of developing distant metastases, improve overall disease control, and potentially can enhance cure rates. If castrate-resistant disease has already emerged then systemic chemotherapy or second-generation anti-androgens should be considered in conjunction with salvage SBRT in order to achieve eradication of such resistant micrometastatic cells, but this needs to be proven in future trials. A similar disparity in the prognosis of castratesensitive versus castrate-resistant patients was shown by Vargas et al. [12] that evaluated salvage brachytherapy in patients with local recurrence following EBRT to the prostate. Five-year biochemical control was $74 \%$ for castrate-sensitive and $22 \%$ for castrate-resistant cases. Pinkawa et al. had previously reported on results of local prostate RT in patients presenting with PSA progression during primary hormonal treatment. The hazard ratio for failure was 7 for patients with CRPC versus those with castrate sensitive disease [28].

Currently, no clear consensus on the optimal management of primary RT failure exists. Salvage radical prostatectomy can be curative in up to $50 \%$ of cases [29-31], but is technically more challenging then primary surgery with higher rates of post-operative complications [31-33], possibly due to radiation-induced fibrosis/adhesions and poorer blood supply and wound healing. Only a small percentage of patients (estimated around $2-3 \%$ ) with primary RT failure will undergo salvage surgery, and most patients with biochemical recurrence following primary $\mathrm{RT}$ are in-fact managed with indefinite ADT monotherapy $[4,5]$. Among the alternative strategies for local salvage of radio-recurrent prostate cancer, the most commonly used method is cryotherapy. Third-generation cryotherapy devices have better accuracy and are employed for focal tumor ablation and thus cause less GI/GU morbidity compared to earlier versions [34]. Randomized controlled studies evaluating the different alternatives for local salvage are lacking and there are no standardized criteria for patient selection. Systematic reviews on local salvage modalities suggest similar efficacy (roughly $50 \%$ long-term biochemical control) with rates of significant toxicity (albeit with differing side-effect profiles) [35, 36]. In a recently published retrospective trial [37] evaluating salvage cryoablation for radio-recurrent prostate cancer approximately $50 \%$ of the patients achieved biochemical salvage with cryoablation at 5 years. Severe complications rates were $3 \%$ for rectal fistula, $7 \%$ for urethral stricture, $25 \%$ for urinary incontinence, and $1 \%$ for death secondary to pulmonary embolism. $\mathrm{Ng}$ et al. [38] assessed the efficacy of salvage cryoablation in 187 patients with locally recurrent prostate cancer after radiotherapy. Patients with pre-cryoablation PSA $<4 \mathrm{ng} / \mathrm{ml}$ had a 5 and 8 -year biochemical RFS of $56 \%$ and $37 \%$, respectively, and patients with pre-cryoablation PSA $\geq 10 \mathrm{ng} / \mathrm{ml}$ had a 5 and 8 -year biochemical recurrence-free survival of only $14 \%$ and $7 \%$, respectively. Ten percent of patients had persistent lower urinary tract symptoms, $3 \%$ severe incontinence and $2 \%$ urethra-rectal fistulas.

Our results are in correspondence with other studies evaluating the role of salvage SBRT for local recurrence after radiation therapy, both in efficacy and toxicity. This is in spite of the fact that patients in our cohort had a much higher risk profile then all previously published studies. For the castrate-sensitive subgroup of our study efficacy was much higher. The skeptic who asks "why use radiation again if it failed first time round?" may be offered an answer based on radiobiological modelling suggesting that ultra-hypofractionation is associated with a different mechanism of action and thus may be more effective.

Leroy et al. [15] retrospectively evaluated salvage SBRT for local recurrence following primary RT in 23 patients. SBRT dose was 36 Gy in 6 fractions. Short-term ADT was initiated in 10 patients, and 4 patients were already on ADT prior to salvage SBRT for biochemical failure. Median follow-up was 22.6 months. Two-year disease-free survival (DFS) was $54 \%$ and median DFS was 27 months. Local DFS at 1- and 2-years were 100\% and $76 \%$. Grade 3 GU cystitis occurred in 2 (9\%) patients and 
1 patient had grade 3 neuralgia. Cumulative grade $2 \mathrm{GU}$ toxicity occurred in 7 patients (30\%), and grade 2 proctitis in $2(9 \%)$ patients.

Pasquier et al. [16] also retrospectively evaluated salvage SBRT for local recurrence after radiation therapy in 100 patients. Median SBRT dose was 36 Gy in 6 fractions. About a third received ADT with SBRT for a median duration of 12 months. Median follow-up was 29 months. Biochemical RFS at 3 years was 55\%. The actuarial 3-year grade $\geq 2$ GU and GI toxicity was $21 \%$ and $1 \%$.

In a study by Fuller et al. 50 patients with local recurrence after prior RT received salvage SBRT [17]. Median follow-up was 44 months. Gleason score at recurrence was $6(n=9), 7(n=22), 8(n=10)$, and $9(n=8)$. SBRT dose was $34 \mathrm{~Gy}$ in 5 fractions. ADT was initiated in 4 patients, and 3 were already on long-term ADT (CRPC). The actuarial 2- and 5-year biochemical RFS rates were $76 \%$ and $60 \%$. The 5 -year actuarial grade $\geq 2$ and grade $\geq 3 \mathrm{GU}$ toxicities were $17 \%$ and $8 \%$, respectively. No acute or late GI toxicity grade $>1$ occurred.

Our study has several limitations. First, the cohort is relatively small and though we have treated over 60 patients to date, we confined the analysis to patients who had at least 2 years of meticulous follow-up. Despite the relatively modest cohort, the difference between castratesensitive and castrate-resistant patients was highly significant, thus generating the hypothesis for more stringent exclusion criteria for aggressive local salvage treatment. About a third of our study cohort had long-standing castrate-resistant disease and most of these patients experienced early distant failure after salvage SBRT. Thus, this study provides some important insights for the success of salvage SBRT including appropriate patient selection and timing of treatment. Secondly, only about two-thirds of patients underwent biopsy for histologic confirmation of local recurrence. In the ideal study all patients should undergo such confirmatory biopsy, however, all patients in our cohort underwent Ga68 PSMA-PET demonstrating local pathologic uptake of PSMA, the positive predictive value (PPV) of which is $99 \%$ in the setting of biochemical recurrence (based on pathologic correlation) according to a recent meta-analysis [39]. The strengths of this study include the availability of PSMA-PET and multi-parametric MRI for early detection of local recurrence. Patient follow-up was meticulous with no patients lost to follow-up. The utilization of ERBs and spacers for rectal sparing, precise image guidance using fiducial markers and central urethral sparing were all important measures to minimize toxicity. Rectal toxicity, specifically bleeding, is a major concern for prostate re-irradiation. To decrease the risk of late rectal toxicity, rectal separation was used all patients. The Hydrogel spacer was inserted between the rectum and prostate before
RT to create a temporary anatomic separation [40], and was shown to decrease the rate of late rectal toxicity after RT to the prostate. A recent study performed on a MRI-Linac also demonstrated a reduction of rotational shifts and intra-fraction motion with the use of a hydrogel spacer [26]. At 3-year follow-up, all grades of rectal bleeding were decreased in patients with hydrogel spacer. ERBs were shown to immobilize the prostate during RT, thereby allowing for reduced treatment margins [41]. In addition, the use of ERBs significantly reduces rectal dose [23] and severe late rectal toxicity [42].

In order to fully evaluate the true potential benefit of salvage SBRT and its impact on the natural history of the disease, future studies should have a more restrictive patient selection. Eligible patients for local salvage attempt should be patients experiencing biochemical failure following primary RT to the prostate, are referred early for advanced imaging such as PSMA-PET demonstrating pathologic local PSMA uptake, which should be confirmed by biopsy, and time from failure to start of salvage SBRT should be as short as possible (early salvage). The use of concomitant ADT with salvage SBRT is an area of debate. Cuccia et al. [43] have reported a series of salvage SBRT in which the majority of patients did not receive ADT with good local control and the benefit of delaying negative impact on quality of life due to castration. We chose to offer a short course of ADT based on a number of assumptions: (a) Androgen depleted cancer cells are more radiosensitive [44]; (b) The addition of ADT to radiotherapy improves PFS and DMFS in recurrent prostate cancer following surgery [44]; (c) A short duration of ADT is associated with a high incidence of testosterone recovery in the vast majority of men [45]; (d) $83 \%$ of our cohort had high risk disease features at recurrence. Duration of concurrent ADT in the setting of salvage SBRT is also an area of debate. Future studies should try to answer whether longer durations of treatment (such as 12 months given in the study by Pasquier [16]) are superior to 6 months of therapy. A recent survey of the Italian Association of Radiotherapy and Clinical Oncology suggests interest in re-irradiation and highlights issues of debate including the irradiated volume, dosimetry parameters, and the use of ADT [46].

\section{Conclusions}

In summary, early delivery of salvage SBRT for local recurrence is associated with excellent 3-year disease control and acceptable toxicity in patients with a castrate-sensitive phenotype. These results appear at the very least comparable to those reported with salvage cryotherapy. Advanced technology for the planning and delivery of salvage re-irradiation with novel measures 
such as rectal spacers and dose painting all contribute to a low rate of serious treatment-related adverse events.

\begin{abstract}
Abbreviations
PC: Prostate cancer; SBRT: Stereotactic body radiation therapy; CRPC: Castrateresistant prostate cancer; PET: Positron emission tomography; PSMA: Prostate specific membrane antigen; PSA: Prostate-specific antigen; Gy: Gray; RFS: Recurrence-free survival; MFS: Metastasis-free survival; GU: Genitourinary; GI: Gastrointestinal; RT: Radiation therapy; SV: Seminal vesicles; HIFU: Highintensity focused ultrasound; ADT: Androgen deprivation therapy; DCE-MRI: Dynamic contrast enhanced magnetic resonance imaging; ERBs: Endo-rectal balloons; EBRT: External-beam radiation therapy; VMAT: Volumetric modulated arc therapy; CTV: Clinical target volume; uPRV: Urethra planning risk volume; CBCT: Cone beam computed tomography; CTCAE: Common terminology criteria for adverse events; NCCN: National comprehensive cancer network; LDR: Low dose rate.
\end{abstract}

\section{Acknowledgements}

Not applicable.

\section{Authors' contributions}

RL and ZS: conception, organization, data analysis, manuscript writing, manuscript editing and final approval. UA: data analysis. ML, RB, and ZD: patient selection for re-irradiation, manuscript editing and final approval. LD, TD, and OP: advanced imaging interpretation, manuscript editing and final approval. LT and MBA: treatment planning, manuscript editing and final approval. IW: patient data collection. All authors read and approved the final manuscript.

\section{Funding}

The authors received no funding for this work.

\section{Availability of data and materials}

The datasets used and/or analyzed during the current study are available from the corresponding author on reasonable request.

\section{Declarations}

\section{Ethics approval}

Ethical approval for this study was obtained from Sheba medical center's IRB ethics ("Helsinki") committee (Approval \#/SMC-9132-11).

\section{Consent for publication}

Not applicable.

\section{Competing interests}

The authors declare that they have no competing interests.

\section{Author details}

'Radiation Oncology Department, Sheba Medical Center, 52621 Ramat-Gan, Israel. ${ }^{2}$ Institute of Urology, Sheba Medical Center, Ramat-Gan, Israel. Institute of Oncology, Sheba Medical Center, Ramat-Gan, Israel. ${ }^{4}$ Sackler School of Medicine, Tel Aviv University, Tel Aviv-Yafo, Israel. ${ }^{5}$ Department of Nuclear Medicine, Sheba Medical Center, Ramat-Gan, Israel. ${ }^{6}$ Department of Radiology, Sheba Medical Center, Ramat-Gan, Israel.

Received: 2 May 2021 Accepted: 10 June 2021

Published online: 23 June 2021

\section{References}

1. Khan MA, Han M, Partin AW, Epstein JI, Walsh PC. Long-term cancer control of radical prostatectomy in men younger than 50 years of age: update 2003. Urology. 2003;62:82-6.

2. Djavan B, Moul JW, Zlotta A, Remzi M, Ravery V. PSA progression following radical prostatectomy and radiation therapy: new standards in the new Millennium. Eur Urol. 2003:43:12-27.
3. Kupelian PA, Mahadevan A, Reddy CA, Reuther AM, Klein EA. Use of different definitions of biochemical failure after external beam radiotherapy changes conclusions about relative treatment efficacy for localized prostate cancer. Urology. 2006;68:593-8. https://doi.org/10.1016/j.urolo gy.2006.03.075.

4. Zumsteg ZS, Spratt DE, Romesser PB, Pei X, Zhang Z, Kollmeier M, et al. Anatomical patterns of recurrence following biochemical relapse in the dose escalation era of external beam radiotherapy for prostate cancer. J Urol. 2015;194:1624-30. https://doi.org/10.1016/j.juro.2015.06.100.

5. Steele EM, Holmes JA. A review of salvage treatment options for disease progression after radiation therapy for localized prostate cancer. Urol Oncol Semin Orig Investig. 2019;37:582-98.

6. Tran H, Kwok J, Pickles T, Tyldesley S, Black PC. Underutilization of local salvage therapy after radiation therapy for prostate cancer. Urol Oncol. 2014;32:701-6.

7. Agarwal PK, Sadetsky N, Konety BR, Resnick MI, Carroll PR. Treatment failure after primary and salvage therapy for prostate cancer: likelihood, patterns of care, and outcomes. Cancer. 2008;112:307-14.

8. Nguyen C, Lairson DR, Swartz MD, Du XL. Risks of major long-term side effects associated with androgen-deprivation therapy in men with prostate cancer. Pharmacotherapy. 2018;38:999-1009.

9. Chandrasekar T, Yang JC, Gao AC, Evans CP. Mechanisms of resistance in castration-resistant prostate cancer (CRPC). Transl Androl Urol. 2015:4:365-80.

10. Crook JM, Zhang P, Pisansky TM, Trabulsi EJ, Amin MB, Bice W, et al. A prospective phase 2 trial of transperineal ultrasound-guided brachytherapy for locally recurrent prostate cancer after external beam radiation therapy (NRG Oncology/RTOG-0526). Int J Radiat Oncol Biol Phys. 2019:103:335-43.

11. National Comprehensive Cancer Network. Prostate Cancer (Version 3.2020). 2020. https://www.nccn.org/professionals/physician_gls/pdf/ prostate.pdf.

12. Vargas C, Swartz D, Vashi A, Blasser M, Kasraeian A, Cesaretti J, et al. Salvage brachytherapy for recurrent prostate cancer. Brachytherapy. 2014;13:53-8.

13. Baumann BC, Baumann JC, Christodouleas JP, Soffen E. Salvage of locally recurrent prostate cancer after external beam radiation using reduceddose brachytherapy with neoadjuvant plus adjuvant androgen deprivation. Brachytherapy. 2017;16:291-8.

14. Yamada Y, Kollmeier MA, Pei X, Kan CC, Cohen GN, Donat SM, et al. A phase II study of salvage high-dose-rate brachytherapy for the treatment of locally recurrent prostate cancer after definitive external beam radiotherapy. Brachytherapy. 2014;13:111-6.

15. Leroy T, Lacornerie T, Bogart E, Nickers P, Lartigau E, Pasquier D. Salvage robotic SBRT for local prostate cancer recurrence after radiotherapy: preliminary results of the Oscar Lambret Center. Radiat Oncol. 2017;12:1-7.

16. Pasquier D, Martinage G, Janoray G, Rojas DP, Zerini D, Goupy F, et al. Salvage stereotactic body radiation therapy for local prostate cancer recurrence after radiation therapy: a retrospective multicenter study of the GETUG. Int J Radiat Oncol Biol Phys. 2019;105:727-34.

17. Fuller D, Wurzer J, Shirazi R, Bridge S, Law J, Crabtree T, et al. Retreatment for local recurrence of prostatic carcinoma after prior therapeutic irradiation: efficacy and toxicity of HDR-like SBRT. Int J Radiat Oncol Biol Phys. 2020;106:291-9.

18. Wondergem M, van der Zant FM, Broos WAM, Knol RJJ. Clinical impact of PSMA PET in biochemically recurrent prostate cancer; a review of the literature. Tijdschr voor Urol. 2020;10:109-21. https://doi.org/10.1007/ s13629-020-00296-6.

19. Kitajima K, Hartman RP, Froemming AT, Hagen CE, Takahashi N, Kawashima A. Detection of local recurrence of prostate cancer after radical prostatectomy using endorectal coil MRI at $3 \mathrm{~T}$ : addition of DWI and dynamic contrast enhancement to T2-weighted MRI. Am J Roentgenol. 2015;205:807-16. https://doi.org/10.2214/AJR.14.14275.

20. Barchetti F, Panebianco V. Multiparametric MRI for recurrent prostate cancer post radical prostatectomy and postradiation therapy. Biomed Res Int. 2014;2014: 316272. https://doi.org/10.1155/2014/316272.

21. Rousseau C, Le Thiec M, Ferrer L, Rusu D, Rauscher A, Maucherat B, et al. Preliminary results of a (68) Ga-PSMA PET/CT prospective study in prostate cancer patients with occult recurrence: diagnostic performance and impact on therapeutic decision-making. Prostate. 2019;79:1514-22. 
22. Patel RR, Orton N, Tomé WA, Chappell R, Ritter MA. Rectal dose sparing with a balloon catheter and ultrasound localization in conformal radiation therapy for prostate cancer. Radiother Oncol. 2003;67:285-94. https://doi.org/10.1016/S0167-8140(03)00056-2.

23. Xiang HF, Lu H-M, Efstathiou JA, Zietman AL, De Armas R, Harris K, et al. Dosimetric impacts of endorectal balloon in CyberKnife stereotactic body radiation therapy (SBRT) for early-stage prostate cancer. J Appl Clin Med Phys. 2017;18:37-43.

24. Karsh LI, Gross ET, Pieczonka CM, Aliotta PJ, Skomra CJ, Ponsky LE, et al. Absorbable hydrogel spacer use in prostate radiotherapy: a comprehensive review of phase 3 clinical trial published data. Urology. 2018;115:3944. https://doi.org/10.1016/j.urology.2017.11.016.

25. Pinkawa M, Escobar Corral N, Caffaro M, Piroth MD, Holy R, Djukic V, et al. Application of a spacer gel to optimize three-dimensional conformal and intensity modulated radiotherapy for prostate cancer. Radiother Oncol. 2011:100:436-41. https://doi.org/10.1016/j.radonc.2011.09.005.

26. Hamstra DA, Mariados N, Sylvester J, Shah D, Karsh L, Hudes R, et al. Continued benefit to rectal separation for prostate radiation therapy: final results of a phase III trial. Int J Radiat Oncol Biol Phys. 2017;97:976-85.

27. Mazzola R, Cuccia F, Figlia V, Giaj-Levra N, Nicosia L, Ricchetti F, et al. New metabolic tracers for detectable PSA levels in the post-prostatectomy setting: is the era of melting glaciers upcoming? Transl Androl Urol. 2019;8(Suppl 5):S538-41.

28. Pinkawa M, Piroth MD, Holy R, Djukic V, Klotz J, Pfister D, et al. Local prostate cancer radiotherapy after prostate-specific antigen progression during primary hormonal therapy. Radiat Oncol. 2012;7:209. https://doi. org/10.1186/1748-717X-7-209.

29. Chade DC, Shariat SF, Cronin AM, Savage CJ, Karnes RJ, Blute ML, et al. Salvage radical prostatectomy for radiation-recurrent prostate cancer: a multi-institutional collaboration. Eur Urol. 2011;60:205-10.

30. Mandel P, Steuber T, Ahyai S, Kriegmair M, Schiffmann J, Boehm K, et al. Salvage radical prostatectomy for recurrent prostate cancer: verification of European Association of Urology guideline criteria. BJU Int. 2016;117:55-61.

31. Sanderson KM, Penson DF, Cai J, Groshen S, Stein JP, Lieskovsky G, et al. Salvage radical prostatectomy: quality of life outcomes and longterm oncological control of radiorecurrent prostate cancer. J Urol. 2006;176:2022-5.

32. Mohler JL, Halabi S, Ryan ST, Al-Daghmin A, Sokoloff MH, Steinberg $\mathrm{GD}$, et al. Management of recurrent prostate cancer after radiotherapy: long-term results from CALGB 9687 (Alliance), a prospective multiinstitutional salvage prostatectomy series. Prostate Cancer Prostatic Dis. 2019;22:309-16.

33. Ward JF, Sebo TJ, Blute ML, Zincke H. Salvage surgery for radiorecurrent prostate cancer: contemporary outcomes. J Urol. 2005;173:1156-60.

34. da Silva RD, Kim FJ. Prostate cancer-local treatment after radiorecurrence: salvage cryoablation. Int Braz J Urol. 2018;44:435-9.

35. Tetreault-Laflamme A, Crook J. Options for salvage of radiation failures for prostate cancer. Semin Radiat Oncol. 2017;27:67-78.
36. Philippou Y, Parker RA, Volanis D, Gnanapragasam VJ. Comparative Oncologic and toxicity outcomes of salvage radical prostatectomy versus nonsurgical therapies for radiorecurrent prostate cancer: a meta-regression analysis. Eur Urol Focus. 2016;2:158-71.

37. Finley D. Salvage cryoablation for radiorecurrent prostate cancer: initial experience at a regional health care system. Perm J. 2019;23:18-153.

38. Ng CK, Moussa M, Downey DB, Chin JL. Salvage cryoablation of the prostate: followup and analysis of predictive factors for outcome. J Urol. 2007;178:1253-7.

39. Hope TA, Goodman JZ, Allen IE, Calais J, Fendler WP, Carroll PR. Metaanalysis of (68)Ga-PSMA-11 PET accuracy for the detection of prostate cancer validated by histopathology. J Nucl Med. 2019;60:786-93.

40. Hatiboglu G, Pinkawa M, Vallée J-P, Hadaschik B, Hohenfellner M. Application technique: placement of a prostate-rectum spacer in men undergoing prostate radiation therapy. BJU Int. 2012;110(11 Pt B):E647-52.

41. McGary JE, Teh BS, Butler EB, Grant W 3rd. Prostate immobilization using a rectal balloon. J Appl Clin Med Phys. 2002;3:6-11.

42. Teh BS, Lewis GD, Mai W, Pino R, Ishiyama H, Butler EB. Long-term outcome of a moderately hypofractionated, intensity-modulated radiotherapy approach using an endorectal balloon for patients with localized prostate cancer. Cancer Commun (Lond, Engl). 2018;38:11.

43. Cuccia F, Mazzola R, Nicosia L, Giaj-Levra N, Figlia V, Ricchetti F, et al. Prostate re-irradiation: current concerns and future perspectives. Expert Rev Anticancer Ther. 2020;20:947-56.

44. Carrie Dr C, Hasbini MDA, de Laroche MDG, Richaud MDP, Guerif MDS, Latorzeff MDI, et al. Salvage radiotherapy with or without short-term hormone therapy for rising prostate-specific antigen concentration after radical prostatectomy (GETUG-AFU 16): a randomised, multicentre, openlabel phase 3 trial. Lancet Oncol. 2016;17:747-56. https://doi.org/10.1016/ S1470-2045(16)00111-X.

45. Forsthoefel MK, Burlile J, Lane SA, Tsou H, Kataria S, Danner M, et al. Testosterone recovery following short course androgen deprivation therapy and stereotactic body radiotherapy correlates closely with improvements in patient-reported hormonal and sexual domain scores. Int J Radiat Oncol Biol Phys. 2019;105:E594-E594. https://doi.org/10.1016/j.ijrobp. 2019.06.1194.

46. Zerini D, Jereczek-Fossa BA, Ciabattoni A, Mirri A, Bertoni F, Fersino S, et al. PROLAPSE: survey about local prostate cancer relapse salvage treatment with external beam re-irradiation: results of the Italian Association of Radiotherapy and Clinical Oncology (AIRO). J Cancer Res Clin Oncol. 2020;146:2311-7. https://doi.org/10.1007/s00432-020-03297-5.

\section{Publisher's Note}

Springer Nature remains neutral with regard to jurisdictional claims in published maps and institutional affiliations.
Ready to submit your research? Choose BMC and benefit from:

- fast, convenient online submission

- thorough peer review by experienced researchers in your field

- rapid publication on acceptance

- support for research data, including large and complex data types

- gold Open Access which fosters wider collaboration and increased citations

- maximum visibility for your research: over $100 \mathrm{M}$ website views per year

At BMC, research is always in progress.

Learn more biomedcentral.com/submissions 